\title{
Using Facial Gestures to Drive Narrative in VR
}

\author{
I. Mavridou \\ Centre of Digital Entertainment \\ \& Emteq Ltd. \\ BH12 5BB, UK \\ imavridou@bournemouth.ac.uk \\ A. Cleal \\ Emteq Ltd, \\ Sussex Innovation Centre, \\ BN1 9SB, UK \\ andrew@emteq.net
}

\author{
M. Hamedi \\ Emteq Ltd, \\ Sussex Innovation Centre, \\ BN1 9SB UK \\ mahyar@emteq.net \\ E. Balaguer-Ballester \\ Faculty of Science and \\ Technology, \\ BH12 5BB, UK \\ eb-ballester@bournemouth.ac.uk
}

\begin{abstract}
We developed an exploratory VR environment, where spatial features and narratives can be manipulated in real time by the facial and head gestures of the user. We are using the Faceteq prototype, exhibited in 2017, as the interactive interface. Faceteq consists of a wearable technology that can be adjusted on commercial HMDs for measuring facial expressions and biometric responses. Faceteq project was founded with the aim to provide a human-centred additional tool for affective humancomputer interaction. The proposed demo will exhibit the hardware and the functionality of the demo in real time.
\end{abstract}

\section{CCS CONCEPTS}

- Human-centered computing $\rightarrow$ Human computer interaction; Interaction paradigms; Virtual reality

\section{KEYWORDS}

Virtual Reality; Facial Expression; Emotion; EMG; Affective Computing

\section{ACM Reference format:}

Mavridou Ifigeneia, Hamedi Mahyar, Fatoorechi Mohsen, Archer James, Cleal Andrew, Balagues-Ballester Emili, Seiss Ellen, and Nduka Charles. 2017. Using Facial Gestures to Drive Narrative in VR. In Proceedings of SUI '17, Brighton, United Kingdom, October 16-17, 2017, 1 pages.

DOI: $10.1145 / 3131277.3134366$

\section{Demo Description}

We designed a VR exploratory environment, consisting of a labyrinth and rooms where the user can explore in real-time. The space unravels secret passages to submerge the viewer deeper into the labyrinth. The player's movement is determined by the user's intention to approach or withdraw where the designated spaces are becoming available to explore based on the user's emotional expressions (e.g. smile, frown). The overall interaction is based solely on facial and head gestural control. Attendees will be able to use facial expressions and head movements as an input modality and react to the demo's VR content. Additionally, we

Permission to make digital or hard copies of part or all of this work for personal or classroom use is granted without fee provided that copies are not made or distributed for profit or commercial advantage and that copies bear this notice and the full citation on the first page. Copyrights for third-party components of this work must be honored. For all other uses, contact the Owner/Author.

SUI '17, October 16-17, 2017, Brighton, United Kingdom

(C) 2017 Copyright is held by the owner/author(s).

ACM ISBN 978-1-4503-5486-8/17/10.

https://doi.org/10.1145/3131277.3134366

\section{Fatoorechi}

Emteq Ltd,

Sussex Innovation Centre, BN1 9SB, UK

mohsen@emteq.net

Faculty of Science and Technology,

BH12 5BB, UK

eseiss@bournemouth.ac.uk

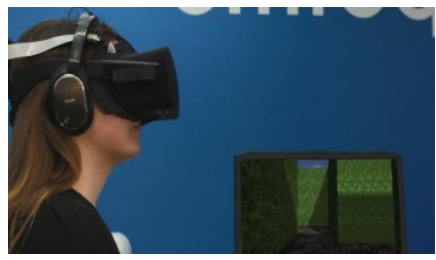

Figure 1: User exploring the demo

\section{E. Seiss}

\author{
J. Archer \\ Emteq Ltd, \\ Sussex Innovation Centre, \\ BN1 9SB, UK \\ james.archer@emteq.net

\section{Nduka \\ Emteq Ltd,} \\ Sussex Innovation Centre, \\ BN1 9SB, UK \\ charles@emteq.net
}

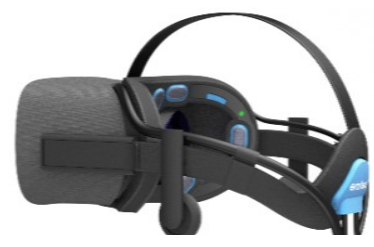

Figure 2: Faceteq integrated on Oculus CV1 will demonstrate how the Faceteq system works to interested attendees.

\section{System Description}

Faceteq is a wearable platform that can be adjusted on commercial head-mounted displays (HMDs) (Figure 1). It utilises EMG technology for muscle tone detection to determine facial muscle activations. This version includes integrated 9DOF accelerometer-gyroscope and photo-plethysmograph (PPG) pulse rate sensors and hence provides movement, posture and physiological state analysis, live data streaming to Unity3D via API, real-time signal quality monitoring and supports ASCII data files as well as binary files for post- acquisition data analysis.

Benefits of measuring facial EMG, can potentially be used: a) as feedback in the design process, b) to measure involuntary emotional reactions at key product purchase evaluation stages, c) to improve user experience during gameplay and d) improve accessibility to those unable to use hand controllers.

\section{Laboratory Description}

The Faceteq platform and the demonstrations were developed by Emteq Ltd, at Innovation Centre, Sussex University, UK. Emteq's vision is to deliver Virtual Reality that can respond to emotional state, by developing facial wearables that can read and interpret facial gestures and arousal states, using a combination of biometric sensors and artificial intelligence techniques.

The company is working closely with scientists and researchers on numerous projects concerning a) Emotion Recognition for naturalistic emotion expression, b) Performance monitoring and drowsiness detection and c) Medical treatment; by developing technology solutions to enable intervention for healthcare.

\section{ACKNOWLEDGEMENTS}

This work is part of a UK EPSRC and Emteq funded research, Centre of Digital Entertainment, EPSRC award: EP/L016540/1. 